ARTÍCULO ORIGINAL

\title{
Efecto de ácidos grasos politinsaturados y ácido nordihidroguaiarético sobre alteraciones pancreáticas en un modelo experimental de diabetes mellitus tipo 2
}

Gustavo Tomás Díaz-Gerevini ${ }^{1,2}$, Jhimy Terceros-Arispe¹, Cristina Beatriz López¹, Lucía Poggio², Alejandro Daín ${ }^{1}$ y Gastón Repossi1*

${ }^{1}$ Histología y Embriología, Facultad de Ciencias Médicas, Instituto de Biología Celular, Universidad Nacional de Córdoba, Córdoba; ${ }^{2}$ Comité de Gerontología, Sociedad Argentina de Diabetes, Buenos Aires. Argentina

\section{RESUMEN}

Se estudiaron los efectos de los ácidos grasos poliinsaturados (PUFA) omega-3 ( $\omega-3)$ y omega-6 ( $\omega-6)$ y el ácido nordehidroguaiarético (NDGA) sobre alteraciones metabólicas y pancreáticas en ratas de la cepa espontánea Stilman-Salgado (e-SS), un modelo espontáneo de diabetes tipo 2. Los animales recibieron mensualmente PUFA y NDGA por vía intraperitoneal. Se estudiaron marcadores metabólicos, de estrés oxidativo e inflamación. Al mes 12 se sacrificaron los animales y se realizaron estudios histopatológicos de páncreas. Las ratas diabéticas e-SS mostraron parámetros alterados en comparación con los controles Wistar no diabéticos. Los tratamientos ensayados modificaron los valores de los marcadores estudiados, el grupo tratado con $\omega-3+$ NDGA mejoró significativamente algunos marcadores compara-

\section{ABSTRACT}

We studied the effects of polyunsaturated fatty acids (PUFAs) omega-3 ( $\omega-3)$ and omega-6 ( $\omega-6)$ and nordehydroguaiaretic acid (NDGA) on metabolic and pancreatic alterations in Stilmann-Salgado (e-SS) rats, a spontaneous model of type 2 diabetes. The animals received monthly PUFAs and NDGA intraperitoneally. Metabolic markers, oxidative stress and inflammation were studied. At month 12 the animals were sacrificed and histopathological studies of the pancreas were performed. The e-SS diabetic rats showed altered parameters compared with non-diabetic Wistar controls. The tested treatments modified the values of the studied markers; the $\omega-3+$ NDGA treated group significantly improved some markers compared with the untreated controls (basal insulin: 0.30 vs. $0.56 \mathrm{U} / \mathrm{ml}$; C-reactive protein: 76 vs. $34 \mathrm{mg} / \mathrm{l}$; 
do con los controles no tratados (insulinemia: 0.30 vs. $0.56 \mathrm{U} / \mathrm{ml}$; proteína $\mathrm{C}$ reactiva: 76 vs. $34 \mathrm{mg} / \mathrm{l}$; interleucina- 6 plasma: 0.273 vs. $0.164 \mathrm{ng} / \mathrm{dl}$; gamma glutamiltranspeptidasa en plasma: 64.6 vs. $42.5 \mathrm{U} / \mathrm{mg}$ proteínas). En el grupo $\omega-3+$ NDGA se observaron menores cambios morfológicos que en los páncreas de ratas diabéticas sin tratar, se redujo la fibrosis $(10.5$ al $4.5 \%$ ) y la frecuencia de atrofia de islotes ( 81.3 al $50 \%$ ) y hemosiderosis (84.4 a $50 \%)$. Las ratas e-SS presentaron cambios en su morfología pancreática acompañadas de altos niveles de marcadores metabólicos, de estrés oxidativo e inflamación. El tratamiento con $\omega-3+$ NDGA mostró mejor capacidad de prevenir/reparar las alteraciones en los parámetros estudiados.

Palabras clave: Diabetes mellitus tipo 2. Ácidos grasos poliinsaturados. Ácido nordihidroguaiarético. Páncreas. Ratas e-SS. plasmatic interleukin-6: 0.274 vs. 0.164 ng/dl; plasmatic gamma-glutamyl transpeptidase: 64.6 vs. $42.5 \mathrm{U} / \mathrm{mg}$ prot). The pancreas of $\omega-3+$ NDGA rats showed less morphological changes than those of untreated diabetic rats, reducing fibrosis (10.5 to $4.5 \%$ ) and decreasing the frequency of islet atrophy ( 81.3 to $50 \%$ ) and hemosiderosis ( 84.4 to $50 \%$ ). The e-SS rats showed changes in their pancreatic morphology accompanied by high levels of metabolic markers, oxidative stress and inflammation. Treatment with $\omega-3+$ NDGA showed better ability to prevent/repair alterations in the parameters studied. (Rev ALAD. 2019;9:59-71)

Corresponding author: Gastón Repossi, grepossi@fcm.unc.edu.ar

Key words: Type 2 diabetes mellitus. Polyunsaturated fatty acids. Nordihydroguaiaretic acid. Pancreas. e-SS rats.

\section{INTRODUCCIÓN}

La diabetes mellitus tipo $2(\mathrm{DM} 2)$ se ha enfocado habitualmente como una enfermedad que afecta al funcionamiento de la porción endocrina del páncreas. Esta visión, ampliamente difundida, considera al páncreas endocrino y exocrino como dos entidades separadas. Sin embargo deberían considerarse como una unidad desde el punto vista morfológico, embriológico, funcional y patológico'. La subdivisión didáctica artificial del páncreas excluye la comprensión del páncreas endocrino-exocrino como un sistema sinérgico interdependiente ${ }^{2,3}$. En seres humanos con síndrome cardiometabólico y DM2 se observó debido a procesos de fibrosis la pérdida de la interfase exocrina periislote-islote, alteraciones en la zona endoacinar y en el intersticio

periacinar interlobular que pueden llevar a la insuficiencia pancreática ${ }^{3,4}$.

En la DM2 existe un proceso de inflamación crónica de bajo grado que se ve reflejada en los elevados niveles de citocinas proinflamatorias como el factor de necrosis tumoral alfa (TNF- $\alpha$ ), la interleucina- 6 (IL-6) y la proteína $C$ reactiva $(P C R)^{5,6}$. Además, en la $\mathrm{DM}_{2}$ hay un incremento del estrés oxidativo y compuestos proapoptósicos, como diversas ceramidas 7 , asociados al desarrollo de lipotoxicidad e inflamación de las células beta y acinos pancreáti$\cos ^{8}$. Estos mecanismos fisiopatogénicos terminarían ocasionando hipoinsulinemia debido a la desdiferenciación o muerte de las células beta9,10.

Existe abundante evidencia epidemiológica, clínica y experimental de que algunos nutrientes, como los 
ácidos grasos $\omega-3$ y antioxidantes, pueden ayudar a prevenir y reparar los daños tisulares producidos por la $\mathrm{DM} 2^{11-13}$. Estos nutrientes han demostrado la capacidad de proteger a las células beta pancreáticas en estas condiciones fisiopatológicas ${ }^{13,14}$.

Los ácidos grasos poliinsaturados (PUFA) de las familias $\omega-3$ y $\omega-6$ son componentes esenciales de la alimentación para los seres humanos, y son precursores, mediante su oxidación por enzimas ciclooxigenasas y lipooxigenasas, de lípidos intensamente bioactivos. Estos metabolitos tienen actividad proinflamatoria y antiinflamatoria: eicosanoides, leucotrienos, endocanabinoides y lipoxinas ${ }^{15,16}$. EI ácido nordehidroguaiarético (NDGA), un antioxidante obtenido del arbusto autóctono jarilla (Larrea sp.), inhibe las enzimas lipooxigenasas y en menor medida las ciclooxigenasas, pudiendo así modular algunos procesos inflamatorios ${ }^{17,18}$.

El objetivo de este trabajo es evaluar los efectos de la administración de PUFA ( $\omega-3$ y $\omega-6)$ y NDGA sobre el metabolismo y morfología pancreáticas en un modelo experimental de DM2.

\section{MATERIALES Y MÉTODOS}

\section{Consideraciones éticas}

Todos los procedimientos con animales fueron aprobados por el comité local de cuidado de animales (CICUAL, Facultad de Ciencias Médicas, Universidad Nacional de Córdoba, Argentina) y se desarrollaron de acuerdo con las normas internacionales.

\section{Animales}

En este trabajo se utilizó como modelo animal experimental de diabetes a ratas de la cepa espontánea Stilman-Salgado (e-SS), que son una línea estable derivada por endocría de ratas Wistar en la
Facultad de Medicina de la Universidad de Rosario. Estos animales desarrollan espontáneamente desde los 6 meses de edad una forma moderada de diabetes no relacionada con la obesidad, que se caracteriza por presentar una rápida intolerancia a la glucosa. La progresión es más severa en los machos y se agrava a medida que envejecen ${ }^{19}$. Los valores normales de los marcadores metabólicos en este modelo son similares a los humanos ${ }^{19}$. En las ratas e-SS se han informado niveles sistémicos elevados de estrés oxidativo y marcadores inflamatorios $^{20}$, así como el desarrollo de alteraciones pancreáticas y encefalopatía diabética ${ }^{21,22}$.

\section{Diseño experimental}

Se utilizaron 140 ratas machos: 120 e-SS diabéticos y 20 Wistar como controles no diabéticos. Los animales fueron criados en el bioterio del Instituto de Biología Celular, Universidad Nacional de Córdoba. Todos los animales recibieron una dieta equilibrada (CHOW rata/ratón, GEPSA, Argentina) y agua, ambos ad-libitum.

Las ratas e-SS fueron asignadas aleatoriamente a los 5 grupos experimentales (Fig. 1). Como tratamiento experimental se administró mensualmente, desde el $2 .^{\circ}$ mes de vida, por vía intraperitoneal $0.4 \mathrm{ml}$ de solución salina isotónica con $0.5 \%$ de etanol (a fin de aumentar la solubilidad de los PUFA) y $6.25 \mathrm{mg} / \mathrm{kg}$ de ácido araquidónico (AA) enriquecido al 90\% (grupos $\omega-6$ ) o $6.25 \mathrm{mg} / \mathrm{kg}$ de aceite de pescado enriquecidos con ácido eicosapentaenoico (EPA) (35\%) y ácido docosahexaenoico (DHA) (40\%) (grupos $\omega-3$ ). En los grupos tratados con NDGA se adicionaron $1.9 \mathrm{mg} / \mathrm{kg}$ de este. En los grupos control se administró solo el vehículo en la misma cantidad y frecuencia que en los tratamientos. Las dosis y la forma de administración han sido probadas y publicadas previamente en modelos murinos de síndrome metabólico y diabetes ${ }^{16,18}$. 

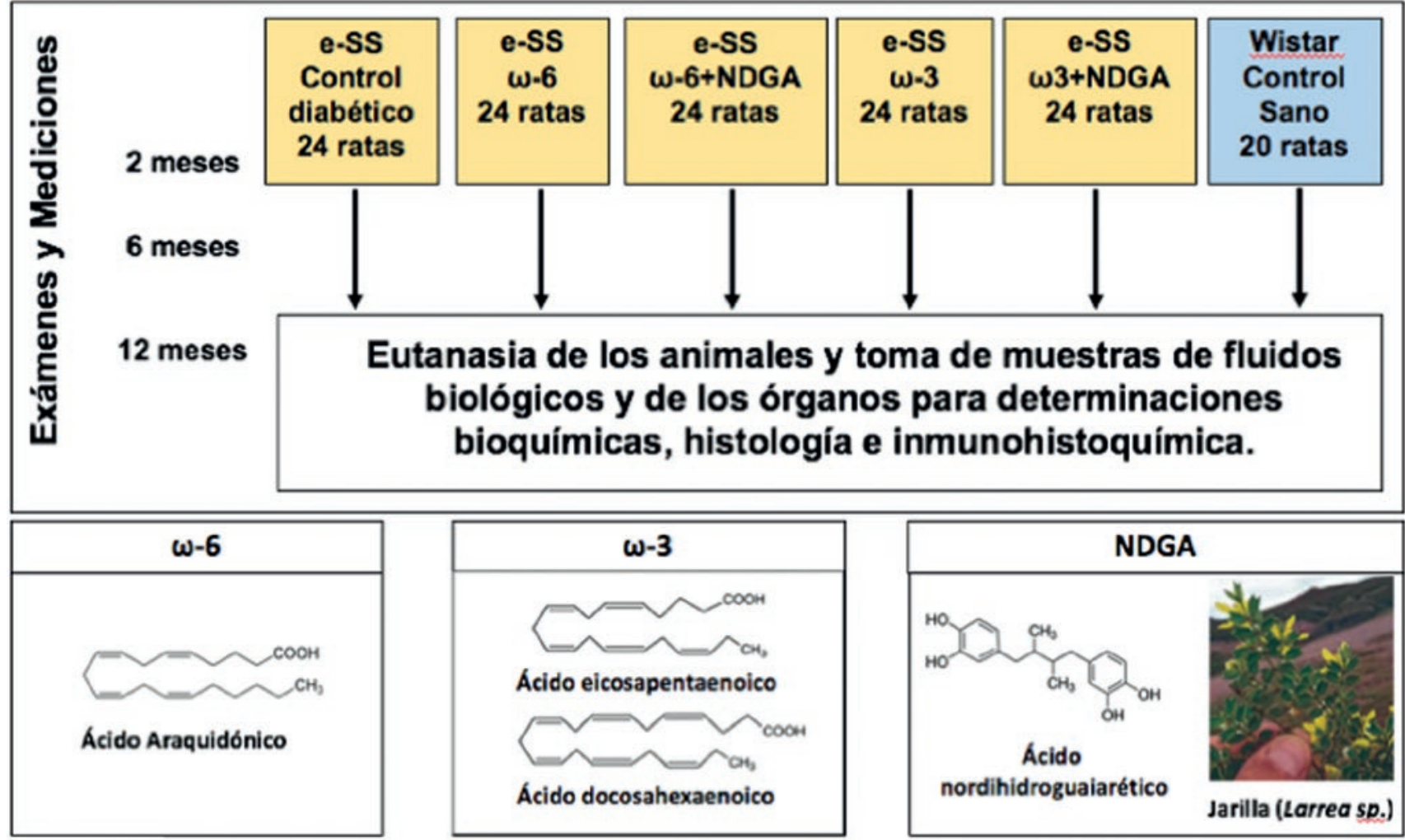

Figura 1. Diseño experimental, grupos de animales y tratamientos experimentales ensayados. e-SS: cepa espontánea Stilman-Salgado; NDGA: ácido nordehidroguaiarético.

\section{Reactivos}

Los reactivos utilizados fueron de calidad analítica, obtenidos comercialmente de los siguientes laboratorios: AA, NDGA y naranja de xilenol de SigmaAldrich $^{\circledast}$ (EE.UU.); aceite de pescado enriquecido con EPA y DHA de Natufarma Labs (Buenos Aires, Argentina). La glucemia y hemoglobina glucosilada (HbA1c) se midieron en sangre capilar mediante sensores Accu-Check Performa ${ }^{\circledR}$ (Roche, Suiza) y A1CNow ${ }^{\circledR}$ (PTS Diagnostics, EE.UU.); kit para determinación de insulina en plasma de ALPCO (EE.UU.). El colesterol total, triglicéridos, $P C R$, enzimas transaminasas, ácido oxalacético pirúvico, oxalacético ácido glutámico y gamma glutamiltranspeptidasa (GGTP) en plasma se determinaron mediante kits de diagnóstico del laboratorio Wiener Lab (Argentina). Los ácidos grasos en plasma se identificaron mediante cromatografía de gases (CG) utilizando un estándar comercial de Nu-check ${ }^{\circledR}$ (EE.UU.). Los solventes y colorantes para histología se obtuvieron de Biopur Diagnostics (Argentina). Los niveles de IL-6 se determinaron con un kit comercial de BD Biosciences (EE.UU.).

\section{Obtención de las muestras}

Semanalmente se pesó a los animales en condiciones estándar y se obtuvo una gota de sangre capilar punzando el extremo distal de la cola para controlar las glucemias en ayunas. A los 12 meses de edad los animales fueron sacrificados mediante sobredosis de isofluorano. Se obtuvo la sangre total, que fue centrifugada a 1,000 $\mathrm{G}$ durante diez minutos, y el plasma utilizado para las determinaciones 
bioquímicas. Luego se procedió a la autopsia sistemática y obtención de material para su evaluación histopatológica, también se conservaron a $-80{ }^{\circ} \mathrm{C}$ muestras de los órganos para determinar el estrés oxidativo y los marcadores inflamatorios en los tejidos. Estas muestras luego fueron homogeneizadas con $1 \mathrm{ml}$ de Buffer fosfato salino (PBS) en un homogeneizador vidrio-teflón y se determinó fotocolorimétricamente su concentración total de proteínas con el método de Bradford, a fin de normalizar las mediciones de los distintos marcadores.

\section{Determinaciones bioquímicas}

Se realizaron las determinaciones de los niveles en plasma de los siguientes biomarcadores:

- Marcadores metabólicos

- Glucemia en ayunas. Luego de ayuno de diez horas, se midió la glucemia con un equipo Accu-Chek Performa ${ }^{\circledR}$ de Roche Diagnostics.

- Glucemia poscarga. Se establecieron los valores glucémicos en ayunas y luego de dos horas de la administración oral de una carga de dos gramos de glucosa/kg de peso ${ }^{10}$.

- HbA1c. Se midió en sangre total mediante un equipo $\mathrm{AC}_{1} \mathrm{Now}^{\circledR}$ de PTS Diagnostics.

- Estudio de los lípidos. Se determinaron los valores de colesterol total y triglicéridos, con kits colorimétricos del laboratorio Wiener (Wiener Labs 1220110 y 1780101).

- Insulinemia. Se midió el valor en el $12 .^{\circ}$ mes, mediante técnica habitual de laboratorio de inmunoensayo de quimioluminiscencia (ALPCO 80-INSMR-CH10). La insulina en la muestra es capturada por inmunoabsorción y se usan anticuerpos conjugados con peroxidasa como reporteros. Se adiciona una solución quimioluminiscente y se lee emisión de luz a 450 nm.
- Marcadores de estrés oxidativo

- Gamma glutamil transpeptidasa (GGTP). Se midieron sus niveles en plasma y en homogenato de tejido pancreático en 10 animales de cada grupo, al $12 .^{\circ}$ mes por fotocolorimetría (Wiener Lab 1421403).

Las enzimas hepáticas, oxalacética-pirúvico y oxalacética-glutámico descarboxilasa se determinaron en plasma por método colorimétrico (Wiener Lab 1751302 y 1761302) a fin de descartar la presencia de daño hepático.

- Hidroperóxidos y lipoperóxidos. Se midieron al mes 12, en plasma mediante la técnica de xylenol-orange (Sigma-Aldrich ${ }^{\circledR}$ 52097) de rutina en nuestro laboratorio'5,22. Las fracciones hidrosolubles y liposolubles de las muestras son aisladas mediante solventes. Los peróxidos oxidan los iones ferrosos (sulfato ferroso amónico) de la solución de reacción formando un complejo coloreado con el naranja de xilenol y su concentración se determina en espectrofotometría a $560 \mathrm{~nm}$.

- Marcadores de inflamación

- PCR ultrasensible. Se midió en plasma, al $12 .^{\circ}$ mes en 10 animales de cada grupo de estudio, mediante la técnica de Turbitest ${ }^{\circledR}$ (Wiener Lab 1683267). La PCR reacciona con un anticuerpo específico formando inmunocomplejos insolubles, la turbidez provocada es proporcional a la concentración de PCR y se determinó espectrofotométricamente a $340 \mathrm{~nm}$.

- IL-6. Cuantificada en plasma de 10 ratas de cada grupo mediante inmuno-ELISA EnzymeLinked ImmunoSorbent Assay) con un kit comercial (BD Biosciences 550319) según el protocolo del proveedor.

- Estudios histopatológicos. En páncreas se realizaron estudios morfológicos y morfométricos 
utilizando tinciones de hematoxilina-eosina (H-E) y Masson.

- H-E y tricrómico de Masson. El páncreas una vez extraído se conservó y procesó para histología. Los cortes se colorearon con H-E y Masson. Las secciones fueron evaluadas utilizando fotomicrografías obtenidas en un microscopio óptico Leika a 40x. Las imágenes se analizaron con el programa Fiji Image J v1.52 $\mathrm{e}^{\circledR}$ (NIH, EE.UU.). Se analizaron preparados histológicos de ocho animales por grupo, cada vidrio contenía cuatro cortes no seriados y se estudiaron dos campos en cada uno de ellos. Parámetros analizados:

- Fibrosis pancreática: incremento del tejido conectivo peri e intraislote, acinar y/o ductal (Fig. 2). Se cuantificó el área ocupada por tejido conectivo en el páncreas utilizando el software Fiji Image $\mathrm{J}^{\circledR}$.

- Atrofia de los islotes de Langerhans: disminución de diámetro, cambios en morfología (forma del islote normal = ovalada; conductos normales $=$ redondeados, epitelio cúbico simple) y fibrosis intraislote.

- Hemosiderosis: presencia agrupada de pigmentos de hemosiderina (Fig. 2).

- Proliferación ductal: más de tres conductos que contactan la parte externa de sus paredes (Fig. 2).

- Nesidioblastosis: aumento de tamaño, deformación y aumento del número celular, con presencia de células anómalas en los islotes de Langerhans (Fig. 2).

\section{Análisis estadístico}

Los resultados se expresaron como media \pm error estándar de la media (SEM). Las comparaciones

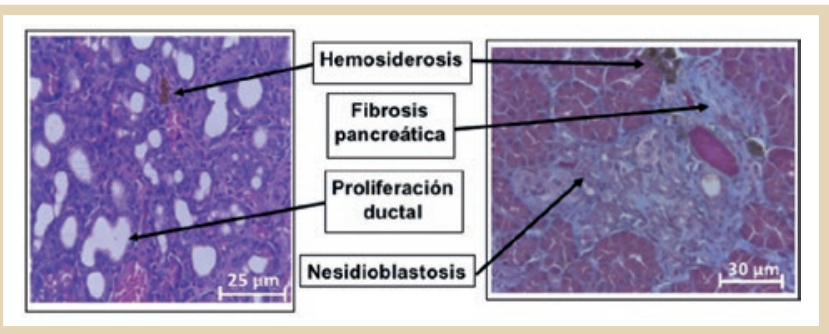

Figura 2. Imágenes ilustrativas de los términos utilizados en el análisis histopatológico.

entre múltiples grupos se analizaron mediante prueba de análisis de la varianza (ANOVA) y se utilizó la prueba $t$ apareada para contrastar los datos de las curvas de peso a diferentes tiempos. La significación estadística para todos los análisis realizados fue $p \leq 0.05$ y se utilizó el programa Infostat ${ }^{\circledR}$ 3.1.

\section{RESULTADOS}

\section{Peso corporal}

En la etapa de posdestete ( 2 meses), las ratas e-SS mostraron un mayor peso (119-66\%) en comparación con el grupo Wistar. Sin embargo, todos los grupos alcanzaron un peso similar al año de estudio (Fig. 3). No se observó obesidad en ninguno de los grupos.

\section{Marcadores metabólicos}

\section{CURVAS GLUCÉMICAS EN AYUNAS $Y$ POSCARGA}

En las ratas e-SS se observó glucemia en ayunas y poscarga normal a los dos meses de edad y valores anómalos, compatibles con la condición de diabetes a partir del $60^{\circ}$ mes $(\geq 200 \mathrm{mg} / \mathrm{dl}$ ). Dichos valores plasmáticos se mantuvieron elevados hasta el final del experimento a los 12 meses (216-228 mg/dl), no se observaron diferencias entre los tratamientos. 


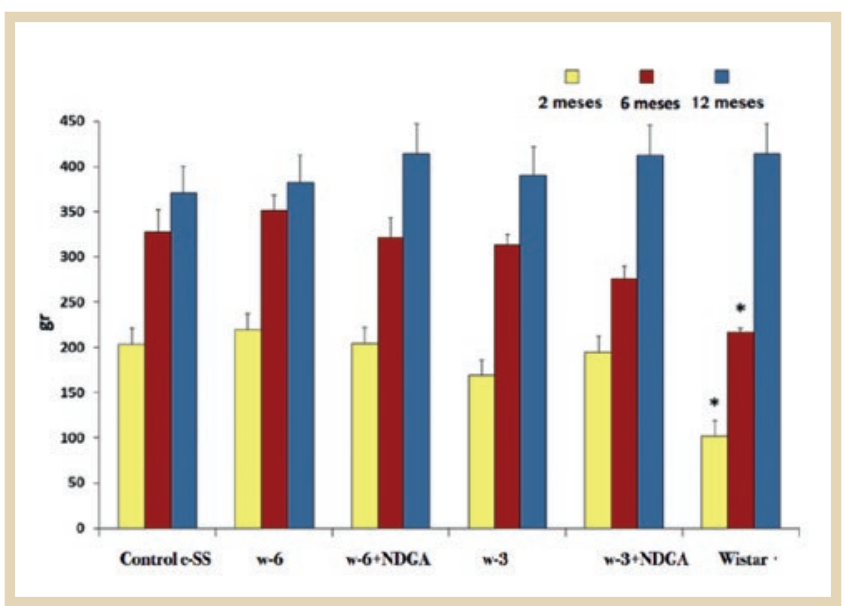

Figura 3. Peso de los animales a los 2,6 y 12 meses. Datos expresados como media \pm SEM. *Diferencia significativa con control e-SS ( $p \leq 0.05$ ). e-SS: cepa espontánea Stilman-Salgado; NDGA: ácido nordehidroguaiarético; SEM: error estándar de la media.

La glucemia en ayunas del grupo Wistar (control no diabético) se mantuvo en rangos normales que oscilaron entre 80 y $100 \mathrm{mg} / \mathrm{dl}$ a lo largo del periodo experimental (Fig. 4).

En las ratas e-SS se registraron valores de HbA1c de 7.7 al $8 \%$ desde el $6 .^{\circ}$ mes hasta finalizar el experimento a los 12 meses, sin diferencias significativas entre los tratamientos.

El grupo Wistar se mantuvo en un rango normal, del $5 \%$ a lo largo de todo el experimento (Tabla 1).

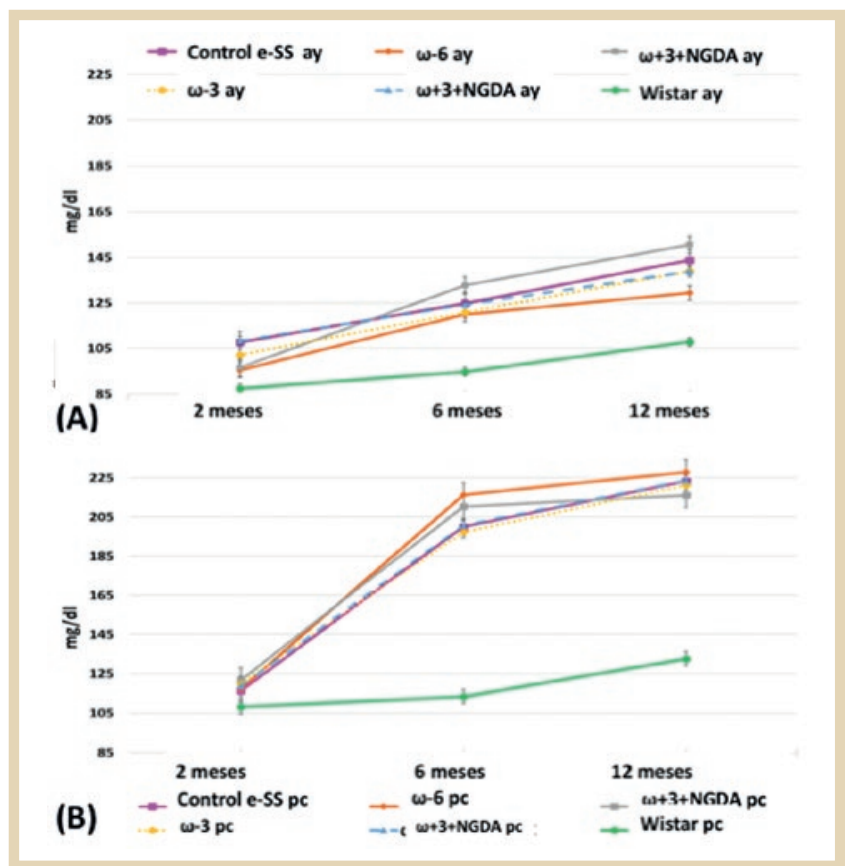

Figura 4. Curva de glucemias en ayunas (A) y poscarga oral (a las 2 h) (B). Datos expresados como media \pm SEM

*Diferencia significativa con control e-SS ( $p \leq 0.05$ ). ay: ayunas; e-SS: cepa espontánea Stilman-Salgado; pc: postcarga de glucosa; SEM: error estándar de la media.

\section{TRIGLICERIDEMIA}

En los animales e-SS desde los 6 meses se detectó hipertrigliceridemia ( $\geq 200 \mathrm{mg} / \mathrm{dl}$ ), manteniéndose elevados los niveles hasta los 12 meses (216-287 mg/dl).

TABLA 1. Valores de marcadores metabólicos e inflamatorios medidos en plasma a los 12 meses. Datos expresados como media \pm SEM de 10 animales por grupo como mínimo

\begin{tabular}{|l|c|c|c|c|c|c|c|}
\hline & $\begin{array}{c}\text { HbA1c } \\
(\%)\end{array}$ & $\begin{array}{c}\text { Trigliceridemia } \\
(\mathrm{mg} / \mathrm{dl})\end{array}$ & $\begin{array}{c}\text { Colesterol } \\
\text { Total }(\mathrm{mg} / \mathrm{dl})\end{array}$ & $\begin{array}{c}\text { Insulina } \\
(\mathrm{U} / \mathrm{ml})\end{array}$ & $\begin{array}{c}\text { CRP } \\
(\mathrm{mg} / \mathrm{L})\end{array}$ & $\begin{array}{c}\text { IL6 Plasma } \\
(\mathrm{ng} / \mathrm{dl})\end{array}$ & $\begin{array}{c}\text { IL6 Páncreas } \\
(\mathrm{ng} / \mathrm{dl})\end{array}$ \\
\hline Control eSS & $8 \pm 0.1$ & $265 \pm 15.6$ & $102.2 \pm 4.6$ & $0.30 \pm 0.03$ & $76 \pm 4.3$ & $0.273 \pm 0.10$ & $0.182 \pm 0.03$ \\
\hline$\omega 6$ & $7.9 \pm 2.4$ & $277.6 \pm 24.0$ & $97.7 \pm 4.7$ & $0.15 \pm 0.22$ & $78 \pm 4.6$ & $0.211 \pm 0.05^{*}$ & $0.177 \pm 0.06$ \\
\hline$\omega 6+$ NDGA & $7.8 \pm 0.3$ & $287.3 \pm 19.4$ & $94.7 \pm 1.6$ & $0.18 \pm 0.10$ & $46 \pm 4.1^{*}$ & $0.198 \pm 0.08^{*}$ & $0.180 \pm 0.04$ \\
\hline$\omega 3$ & $7.8 \pm 0.4$ & $243 \pm 5.5^{*}$ & $104.1 \pm 3.0$ & $0.20 \pm 0.02$ & $47 \pm 3.3^{*}$ & $0.178 \pm 0.11^{*}$ & $0.170 \pm 0.08$ \\
\hline$\omega 3+$ NDGA & $7.7 \pm 0.6$ & $216.8 \pm 4.1^{*}$ & $92.7 \pm 1.4$ & $0.56 \pm 0.16^{*}$ & $34 \pm 3.1^{*}$ & $0.164 \pm 0.06^{*}$ & $0.156 \pm 0.07^{*}$ \\
\hline Wistar & $5 \pm 0.8^{*}$ & $126 \pm 1.5^{*}$ & $110 \pm 2.9$ & $7.96 \pm 0.30^{*}$ & $3 \pm 0.2^{*}$ & $0.156 \pm 0.04^{*}$ & $0.140 \pm 0.01^{*}$ \\
\hline
\end{tabular}

*Indican diferencia significativa con el grupo control e-SS ( $p \leq 0.05)$.

e-SS: cepa espontánea Stilman-Salgado; HbA1c: hemoglobina glucosilada; IL-6: interleucina-6; NDGA: ácido nordehidroguaiarético; PCR: proteína C reactiva; SEM: error estándar de la media. 
En animales diabéticos tratados con $\omega-3$ y $\omega-3+N D G A$ las trigliceridemias fueron significativamente menores (9 y 19\% menos) que en el grupo control e-SS.

El grupo Wistar permaneció durante toda la experiencia en valores normales que oscilaron entre 80 y $126 \mathrm{mg} / \mathrm{dl}$ (Tabla 1 ).

\section{Colesterol tOtAL}

Los valores plasmáticos de colesterol total se mantuvieron en valores que oscilaron entre 92 y $110 \mathrm{mg} / \mathrm{dl}$ en todos los grupos, incluidos los controles no diabéticos, sin diferencias significativas entre ellos (Tabla 1).

\section{INSULINEMIA}

Las ratas e-SS a los 12 meses tienen bajos niveles de insulina en plasma (0.15-0.30 U/ml).

Aquellas ratas e-SS tratadas con $\omega-3+$ NDGA mostraron un aumento (87\%) comparado con los controles sin tratamiento, pero sin llegar a los valores de los controles Wistar (0.56 vs. $7.96 \mathrm{U} / \mathrm{ml}$ ) (Tabla 1).

\section{Marcadores inflamatorios}

\section{Proteína C Reactiva}

En este trabajo se consideró que los valores de esta proteína de fase aguda en las ratas son normales hasta $10 \mathrm{mg} / \mathrm{l}^{6,8}$. En las ratas e-SS se observaron niveles plasmáticos muy elevados (11 a 26 veces) a los 12 meses comparados con los controles no diabéticos.

En los animales tratados con $\omega-3, \omega-3+$ NDGA y $\omega-6+N D G A$ se detectaron niveles menores $(38,56$ y 40\%, respectivamente) de PCR comparado con los animales diabéticos no tratados (Tabla 1).

\section{NIVELES DE INTERLEUCINA-6}

A los 12 meses los valores hallados de IL-6 plasmática (5-75\%) y pancreática (11-30\%) fueron más altos en las ratas diabéticas e-SS que en las Wistar. Todos los tratamientos utilizados disminuyeron los niveles de IL-6 plasmáticos comparado con el grupo control e-SS, mientras que en páncreas solo el tratamiento $\omega-3+N D G A$ logró disminuir la IL-6 (Tabla 1). Las ratas bajo este tratamiento ( $\omega-3+$ NDGA) mostraron en plasma concentraciones de IL-6 similares a los controles no diabéticos.

\section{Marcadores de estrés oxidativo}

\section{ACTIVIDAD DE GAMMA GLUTAMILTRANSPEPTIDASA}

Las ratas e-SS a los 12 meses mostraron niveles de actividad GGTP 6 a 10 veces más altos que los controles no diabéticos (Fig. 5A) en plasma. Los animales tratados con $\omega-3, \omega-3+$ NDGA y $\omega-6+$ NDGA disminuyeron la GGTP plasmática (27, 52 y 73\%, respectivamente) comparados con los animales diabéticos sin tratamiento (control e-SS).

La actividad GGTP detectada en tejido pancreático fue de 2 a 12 veces menor que en plasma. En los animales diabéticos los niveles de GGTP fueron mayores (48-100\%) que en los controles Wistar. Solo el tratamiento con $\omega-3+N D G A$ disminuyó significativamente ( $43 \%$ menos) la actividad GGTP en el tejido pancreático comparado con el grupo control e-SS (Fig. 5A).

\section{Peróxidos}

Las ratas e-SS a los 12 meses tienen altos niveles de peroxidación (13 a 134\% mayores) comparados con los animales Wistar. En el plasma se encuentran principalmente elevados los hidroperóxidos y el 

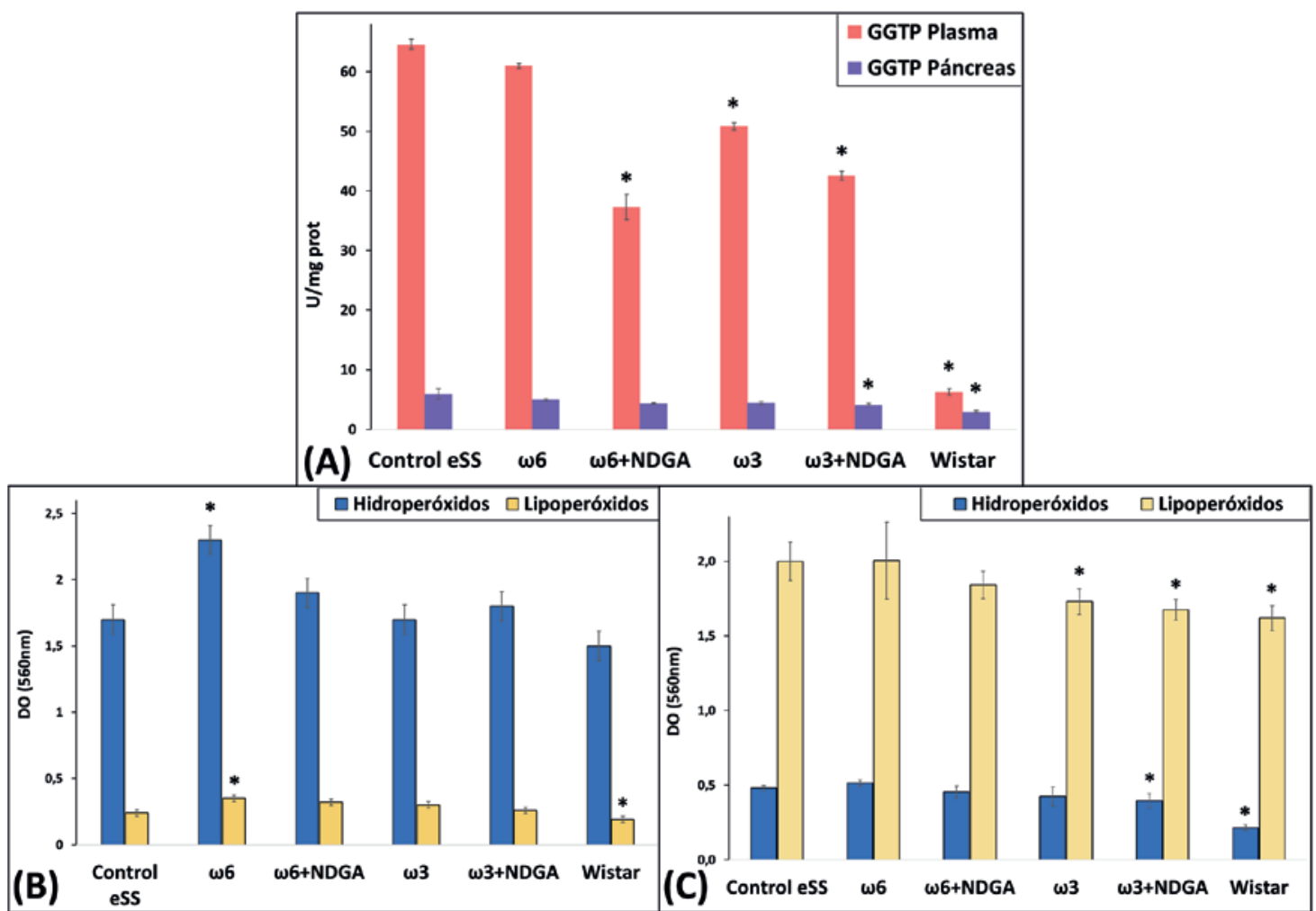

Figura 5. Marcadores de estrés oxidativo en plasma y tejido pancreático de los animales experimentales a los 12 meses. A: actividad específica GGTP. B: peróxidos en plasma. C: peróxidos en tejido pancreático. Datos expresados como media \pm SEM.

*Diferencia significativa con control e-SS ( $p \leq 0.05$ ).

DO: densidad óptica; e-SS: cepa espontánea Stilman-Salgado; SEM: error estándar de la media.

tratamiento con $\omega-6$ los incrementó un 35\% por encima de los valores hallados en los controles no tratados (Fig. 5B). En cambio, en el tejido pancreático los niveles de lipoperóxidos son mayores que los de hidroperóxidos. En los animales tratados con $\omega-3$ y $\omega-3+$ NDGA los lipoperóxidos se redujeron un 15 y $20 \%$ respectivamente, mientras que los hidroperóxidos disminuyeron un $20 \%$ en el grupo $\omega-3+$ NDGA comparado con el control e-SS (Fig. 5C).

\section{Estudios histopatológicos}

\section{FIBROSIS PANCREÁTICA}

Se observó mediante la técnica de Masson que las ratas e-SS presentaron mayor fibrosis ( 1.5 a 3.5 veces más), caracterizada por un aumento del tejido conectivo en todo el páncreas (endocrino/exocrino), comparados con los animales Wistar. En los animales diabéticos tratados con $\omega-6+$ NDGA, $\omega-3$ y $\omega-3+$ NDGA se observaron disminuciones del 52, 63 y $58 \%$ respectivamente de la fibrosis comparado con el grupo control e-SS (Tabla 2).

\section{ATROFIA DE LOS ISLOTES DE LANGERHANS}

En preparados histológicos teñidos con $\mathrm{H}$-E de ratas e-SS los islotes de Langerhans fueron de menor diámetro (47.4\% promedio) que los de ratas Wistar, además presentaron bordes irregulares, forma estrellada, fibrosis intraislote y en algunos casos se observaron estructuras similares a conductos 
Tabla 2. Estudio histopatológico de páncreas en preparados teñidos con hematoxilina-eosina y Masson. La fibrosis se expresa como proporción de área ocupada por tejido conectivo por campo analizado con el software Image ${ }^{\circledR}$

\begin{tabular}{|c|c|c|c|c|c|}
\hline & Fibrosis (\% Área) & Atrofia de islotes & Hemosiderosis & Proliferación Ductal & Nesidioblastosis \\
\hline Control eSS & 10.5 & $\begin{array}{c}++++ \\
(81.3 \%)\end{array}$ & $\begin{array}{c}++++ \\
(84.4 \%)\end{array}$ & $\begin{array}{c}++ \\
(50 \%)\end{array}$ & $\begin{array}{c}+++ \\
(56.3 \%)\end{array}$ \\
\hline$\omega 6$ & 7.8 & $\begin{array}{c}+++ \\
(78.1 \%)\end{array}$ & $\begin{array}{c}+++ \\
(81.3 \%)\end{array}$ & $\begin{array}{c}\stackrel{++}{++} \\
(81.3 \%)^{*}\end{array}$ & $\begin{array}{c}+++ \\
(50 \%)\end{array}$ \\
\hline$\omega 6+N D G A$ & $5.1^{*}$ & $\begin{array}{c}+++ \\
(71.9 \%)\end{array}$ & $\begin{array}{c}\stackrel{++}{++} \\
(68.8 \%)^{*}\end{array}$ & $\begin{array}{c}++ \\
(50 \%)\end{array}$ & $\begin{array}{c}++ \\
(40.6 \%)^{*}\end{array}$ \\
\hline$\omega 3$ & $3.9^{*}$ & $\begin{array}{c}++ \\
(50 \%)^{*}\end{array}$ & $\begin{array}{c}++ \\
(50 \%)^{*}\end{array}$ & $\begin{array}{c}++ \\
(50 \%)\end{array}$ & $\begin{array}{c}+ \\
(31.3 \%)^{*}\end{array}$ \\
\hline$\omega 3+N D G A$ & $4.5^{*}$ & $\begin{array}{c}++ \\
(50 \%)^{*}\end{array}$ & $\begin{array}{c}++ \\
(50 \%)^{*}\end{array}$ & $\begin{array}{c}++ \\
(50 \%)\end{array}$ & $\begin{array}{c}+++ \\
(53.1 \%)\end{array}$ \\
\hline Wistar & $3.0 *$ & -- & --- & -- & --- \\
\hline
\end{tabular}

Las cruces (+) indican el nivel de la alteración histológica analizada y el porcentaje refleja la frecuencia de campos microscópicos examinados en los que se detectó la misma. Los guiones (--) indican que no se observó dicha alteración.

*Indican diferencia significativa con grupo control e-SS ( $\leq \leq 0.05)$.

e-SS: cepa espontánea Stilman-Salgado; NDGA: ácido nordehidroguaiarético.

intercalares dentro del islote (Tabla 2). Los animales diabéticos tratados con $\omega-3$ y $\omega-3+$ NDGA presentaron un $38 \%$ menos de islotes atrofiados comparados con los controles no tratados (control e-SS).

\section{HEMOSIDEROSIS}

Se observó la presencia de hemosiderosis en más del $80 \%$ de los preparados teñidos con $\mathrm{H}$-E, analizados de ratas diabéticas no tratadas (control e-SS), esta proporción fue menor en aquellos animales tratados con $\omega-6+\operatorname{NDGA}(-18 \%), \omega-3(-41 \%)$ y $\omega-3+N D G A(-41 \%)$. En los preparados histológicos de páncreas de animales Wistar no se detectó hemosiderosis (Tabla 2).

\section{PRoliferaCIÓN DUCTAL}

En el tejido pancreático de las ratas diabéticas e-SS se observó un $50 \%$ más de estructuras similares a conductos que en las ratas Wistar. En el grupo de animales tratados con $\omega-6$ la presencia de estas estructuras aumentó hasta más de un 80\% comparado con los controles no diabéticos (Tabla 2).

\section{NESIDIOBLASTOSIS}

En los preparados teñidos con $\mathrm{H}-\mathrm{E}$ de páncreas de ratas e-SS de 12 meses sin tratamiento, grupo control e-SS, se observó la presencia de células con características histológicas anómalas en más del $56 \%$ de los islotes de Langerhans estudiados. En los animales diabéticos tratados con $\omega-6+$ NDGA y $\omega-3$ estas alteraciones son menos frecuentes ( 28 y $44 \%$, respectivamente) que en los islotes pancreáticos de los animales del grupo control e-SS. Estas características anómalas no se observaron en ningún preparado obtenido de los controles Wistar no diabéticos (Tabla 2).

\section{DISCUSIÓN}

Los compuestos, PUFA y NDGA, utilizados como tratamientos mostraron capacidad de modular los 
parámetros estudiados en las ratas e-SS. A nivel bioquímico el tratamiento combinado de PUFA $\omega-3$ más NDGA disminuyó los niveles de trigliceridemia, marcadores inflamatorios y estrés oxidativo, efecto ya observado en trabajos anteriores ${ }^{11-15,17,18,20,22-25}$. Los animales bajo este tratamiento también mostraron un aumento ( $87 \%$ comparado con control e-SS) de la insulinemia, pero aún permanecen muy bajos comparados con los controles no diabéticos. Sin embargo, los tratamientos utilizados no afectaron significativamente los marcadores del metabolismo glucémico. Los grupos de animales diabéticos tratados con PUFA $\omega-3$ fueron los que mostraron menos alteraciones morfológicas pancreáticas (Tabla 2).

La hipertrigliceridemia, un hallazgo frecuente en pacientes con $\mathrm{DM} 2$, también se observó en las ratas e-SS (Tabla 1) con menores concentraciones de PUFA en plasma ${ }^{11,23}$. El aumento de los triglicéridos en $\mathrm{DM} 2$ se asocia a mecanismos de lipotoxicidad que llevan a inflamación, estrés oxidativo ${ }^{24-26}$ y a la progresiva muerte de las células beta por apopto$\mathrm{sis}^{10,27}$. Esto provoca una disminución de la insulinemia y al desarrollo de atrofia pancreática, un proceso similar a lo observado por el grupo de Tarrés ${ }^{19,21}$. En este trabajo, y en anteriores de nuestro laboratorio, observamos que el aporte de $\omega-3+$ NDGA se asoció a una disminución de los valores de triglicéridos plasmáticos ( $22 \%$ vs. control e-SS) en las ratas diabéticas a los 12 meses de edad ${ }^{20,25}$. Los ácidos grasos $\omega$-3 ejercen efectos biológicos, en parte, mediante la activación de los receptores del proliferador activado de peroxisomas que regula la transcripción de los genes implicados en la proliferación, la supervivencia y el metabolismo celu$\operatorname{lar}^{24,28}$. La activación de estos receptores lleva a la fosforilación la vía factor nuclear kappa beta (NF-k $\beta$ ), implicada en la respuesta celular frente al estrés, y a una reducción de la respuesta inflamatoria ${ }^{28}$. La adición de NDGA a los PUFA $\omega-3$ en nuestros ensayos reforzó los efectos beneficiosos del tratamiento. Este compuesto actuaría por dos mecanismos: directamente como antioxidante contrarrestando el estrés oxidativo $0^{17,20}$ e indirectamente inhibiendo enzimas ciclooxigenasas, responsables de la producción de sustancias proinflamatorias, lo cual se manifestaría en menores concentraciones de los marcadores inflamatorios estudiados ${ }^{18,20,25}$. Esta disminución del estrés celular (oxidativo e inflamatorio) podría permitir la prevención/reparación de las alteraciones morfológicas observadas en el tejido pancreático.

Histológicamente se observó una afectación global del páncreas de nuestros animales experimentales diabéticos caracterizada por fibrosis de la porción exocrina y endocrina, hemosiderosis, atrofia de los islotes y proliferación ductal (Tabla 2). En los islotes de Langerhans se encontraron cambios consistentes en aumento del tamaño, número y heterogeneidad celular, similares a los ya descritos por Picena, et al., quienes lo caracterizaron como nesidioblastosis ${ }^{21}$. Esta lesión por definición se asocia a una proliferación de células beta. Sin embargo, ni en trabajos anteriores de otros grupos con ratas e-SS ${ }^{19,21}$ ni en nuestro laboratorio se detectaron incrementos en los niveles de insulina ${ }^{20,22,25}$. En nuestros experimentos en los animales que mostraron este tipo de alteración histológica (Tabla 2) se detectó una disminución marcada de la insulinemia (Tabla 1), lo cual no coincide con la descripción clásica de nesidioblastosis como lesión proliferativa de células beta. Mediante los mecanismos de glucolipotoxicidad se genera estrés oxidativo e inflamación crónica de bajo grado, lo cual lleva a fibrosis tanto en el páncreas endocrino como en el exocrino $8,29,30$.

En las estructuras exocrinas del páncreas se observó fibrosis y proliferación de conductos formando pequeños grupos (Fig. 3), lo cual se ha descrito escasamente con anterioridad. Esta proliferación 
ductal podría estar relacionada con la atrofia tanto de la porción endocrina como exocrina y a los cambios en la secreción de insulina, como un intento por regenerar el parénquima perdido 4,29,30. Este fenómeno podría también estar relacionado con la asociación entre DM2 y adenocarcinoma ductal de páncreas $^{31}$. La insuficiencia pancreática exocrina se demostró en aproximadamente el 50\% de los pacientes con diabetes tipo 1 y en el 30-50\% de los pacientes con $\mathrm{DM}^{29}$. Hallazgos similares fueron descritos por Olsen, et al., quienes reportaron signos de cambios inflamatorios crónicos en el $13 \%$ de los páncreas de 394 autopsias de pacientes diabéti$\cos ^{5}$. Las alteraciones morfológicas del páncreas exocrino/endocrino se han asociado a inflamación crónica de bajo grado, condición en la cual los marcadores inflamatorios como la PCR y la IL-6 predicen el desarrollo de las complicaciones asociadas a $\mathrm{DM}_{2}{ }^{5,6,10}$, y estrés oxidativo ${ }^{32}$. Estas condiciones nocivas estrechamente involucradas en la patogénesis de la DM2 llevarían con el tiempo a la atrofia del órgano4-6,9,12,26,27,29.

Los focos de hemosiderosis observados en el estudio histopatológico podrían deberse a microhemorragias intrapancreáticas ${ }^{33}$. El hierro liberado de los eritrocitos extravasados de lesiones microvasculares se deposita y finalmente aparecen depósitos significativos de hemosiderina asociada a inflamación crónica34. El hierro es bien conocido por ser tóxico para los islotes de las células beta35, con una cantidad creciente de evidencia de que incluso las formas leves de su sobrecarga pueden contribuir a la patogénesis de la $\mathrm{DM} 2^{30-32}$. Estudios epidemiológicos han demostrado una mayor tasa de $\mathrm{DM} 2$ en aquellas poblaciones con niveles más altos (cuartiles o quintiles) de ferritina sérica ${ }^{36}$. En nuestros ensayos estas lesiones morfológicas observadas en el tejido pancreático también se previnieron/repararon en los animales tratados con $\omega-3$ y $\omega-3+$ NDGA.

\section{CONCLUSIONES}

Las ratas e-SS presentaron modificaciones metabólicas, altos niveles de marcadores de estrés oxidativo e inflamación y alteraciones en la morfología del tejido pancreático. Los animales diabéticos tratados con PUFA $\omega-3$ adicionado con NDGA mostraron una mejoría en los parámetros estudiados, con valores más cercanos a los controles no diabéticos (grupo Wistar). Por lo que la utilización de esta combinación de nutrientes puede ser una valiosa herramienta terapéutica en el tratamiento de la diabetes mellitus y sus complicaciones.

\section{AGRADECIMIENTOS}

Algunos reactivos fueron cedidos por el Prof. Undurti N. Das (República de la India), la Fundación Grupo Amigos de los Diabéticos (Personería Jurídica 231) y la Fundación de la FCM-UNC.

Este trabajo fue solventado con fondos de la Secretaría de Ciencia y Tecnología de la Universidad Nacional de Córdoba y de la SECyT de la Universidad Nacional de La Rioja.

\section{FINANCIAMIENTO}

Este trabajo fue financiado con fondos provistos por la Secretaría de Ciencia y Tecnología de la Universidad Nacional de Córdoba y la Fundación Amigos de los Diabéticos de Villa Carlos Paz, Argentina.

\section{BIBLIOGRAFÍA}

1. Pieler T, Chen Y. Forgotten and novel aspects in pancreas development. Biol Cell. 2006;98:79-88.

2. Jennings RE, Berry AA, Strutt JP, Gerrard DT, Hanley NA. Human pancreas development. Development. 2015;142:3126-37.

3. Bertelli E, Bendayan M. Association between endocrine pancreas and ductal system. More than an epiphenomenon of endocrine differentiation and development? J Histochem Cytochem. 2005;53: 1071-86. 
4. Hayden MR, Patel K, Habibi J, Gupta D, Tekwani SS, Whaley-Connell $A$, et al. Attenuation of endocrine-exocrine pancreatic communication in type 2 diabetes: pancreatic extracellular matrix ultrastructural abnormalities. J Cardiometab Syndr. 2008;3:234-43.

5. Olsen TS. The incidence and clinical relevance of chronic inflammation in the pancreas in autopsy material. Acta Pathol Microbiol Scand A. 1978;86:361-5.

6. Fuse M, Yokoi N, Shinohara M, Masuyama T, Kitazawa R, Kitazawa S, et al. Identification of a major locus for islet inflammation and fibrosis in the spontaneously diabetic Torii rat. Physiol Genomics. 2008;35: 96-105.

7. Karunakaran U, Elumalai S, Moon JS, Won KC. CD36 dependent redoxosomes promotes ceramide-mediated pancreatic $\beta$-cell failure via p66Shc activation. Free Radic Biol Med. 2019;134:505-15.

8. Hameed I, Masoodi SR, Mir SA, Nabi M, Ghazanfar K, Ganai BA. Type 2 diabetes mellitus: From a metabolic disorder to an inflammatory condition. World J Diabetes. 2015;6:598-612.

9. Talchai C, Xuan S, Lin HV, Sussel L, Accili D. Pancreatic $\beta$-cell dedifferentiation as mechanism of diabetic $\beta$-cell failure. Cell. 2012;150:122334.

10. Tangvarasittichai S. Oxidative stress, insulin resistance, dyslipidemia and type 2 diabetes mellitus. World J Diabetes. 2015;6:456-80.

11. Chen C, Yang Y, Yu X, Hu S, Shao S. Association between omega-3 fatty acids consumption and the risk of type 2 diabetes: A meta-analysis of cohort studies. J Diabetes Investig. 2017;8(4):480-8.

12. Acosta-Montaño P, García-González V. Effects of dietary fatty acids in pancreatic beta cell metabolism, implications in homeostasis. Nutrients. 2018;10(4):393.

13. Dass AS, Narayana S, Venkatarathnamma PN. Effect of vitamin E and omega 3 fatty acids in type 2 diabetes mellitus patients. J Adv Pharm Technol Res. 2018;9(1):32-6.

14. Calder PC. Omega-3 fatty acids and inflammatory processes: from molecules to man. Biochem Soc Trans. 2017;45(5):1105-15.

15. Repossi G, Dain A, Eynard AG. Dietary manipulations of polyunsaturated fatty acids (PUFA) substrates of endocannabinoids: implications in human health and diseases. Curr Nutr Food Sci. 2009;5(2): $112-25$.

16. Gundala NKV, Naidu VGM, Das UN. Arachidonic acid and lipoxin A4 attenuate streptozotocin-induced cytotoxicity to RIN5 F cells in vitro and type 1 and type 2 diabetes mellitus in vivo. Nutrition. 2017;35:65-80.

17. Guzmán-Beltrán S, Pedraza-Chaverri J, Gonzalez-Reyes S, Hernández-Sánchez F, Juarez-Figueroa UE, Gonzalez Y, et al. Nordihydroguaiaretic acid attenuates the oxidative stress-induced decrease of CD33 expression in human monocytes. Oxid Med Cell Longev. 2013;2013: 375893.

18. Chan JKW, Bittner S, Bittner A, Atwal S, Shen WJ, Inayathullah M, et al. Nordihydroguaiaretic acid, a lignan from Larrea tridentata (Creosote Bush), protects against american lifestyle-induced obesity syndrome diet-induced metabolic dysfunction in mice. J Pharmacol Exp Ther. 2018;365(2):281-90.

19. Tarrés MC, Martinez SM, Montenegro S, Llorens A, Picena JC, Naves A. The e-SS rat. A model for non-insulin-dependent human diabetes. Am J Pathol. 1992;141:761-3.

20. Dain A, Repossi G, Diaz-Gerevini GT, Vanamala J, Das UN, Eynard AR. Long chain polyunsaturated fatty acids (LCPUFAs) and nordihydro- guaiaretic acid (NDGA) modulate metabolic and inflammatory markers in a spontaneous type 2 diabetes mellitus model (Stillman Salgado rats). Lipids Health Dis. 2016;15(1):205.

21. Picena JC, Montenegro SM, Tarrés MC, Martínez SM. Modificaciones dinámicas en los islotes de Langerhans de dos líneas de ratas espontáneamente diabéticas. Medicina (B. Aires). 2007;67:331-40.

22. Díaz-Gerevini GT, Repossi G, Dain A, Tarres MC, Das UN, Eynard AR Cognitive and motor perturbations in elderly with longstanding diabetes mellitus. Nutrition. 2014;30(6):628-35.

23. Dain A, Repossi G, Das UN, Eynard AR. Role of PUFAs, the precursors of endocannabinoids, in human obesity and type 2 diabetes. Front Biosci (Elite Ed). 2010;2:1432-47.

24. Perez-Martinez P, Perez-Jimenez F, Lopez-Miranda J. n-3 PUFA and lipotoxicity. Biochim Biophys Acta. 2010;1801:362-6.

25. Díaz-Gerevini GT, Daín A, Pasqualini ME, López CB, Eynard AR, Repossi G. Diabetic encephalopathy: beneficial effects of supplementation with fatty acids $\omega-3$ and nordihydroguaiaretic acid in a spontaneous diabetes rat model. Lipids Health Dis. 2019;18(1):43.

26. Gerber PA, Rutter GA. The role of oxidative stress and hypoxia in pancreatic beta-cell dysfunction in diabetes mellitus. Antioxid Redox Signal. 2017;26(10):501-18.

27. Jiao J, Dou L, Li M, Lu Y, Guo HB, Man Y, et al. NADPH oxidase 2 plays a critical role in dysfunction and apoptosis of pancreatic $\beta$-cells induced by very low-density lipoprotein. Mol Cell Biochem. 2012;370: 103-13.

28. Vitale SG, Laganà AS, Nigro A, La Rosa VL, Rossetti P, Rapisarda AM, et al. Peroxisome proliferator-activated receptor modulation during metabolic diseases and cancers: Master and minions. PPAR Res. 2016; 2016:6517313.

29. Pezzilli R, Andriulli A, Bassi C, Balzano G, Cantore M, Delle Fave G, et al. The Exocrine Pancreatic Insufficiency collaborative (EPIC) Group. Exocrine pancreatic insufficiency in adults: A shared position statement of the Italian association for the study of the pancreas. World J Gastroenterol. 2013;19:7930-46.

30. Piciucchi M, Capurso G, Archibugi L, Delle Fave M, Capasso M, Delle Fave G. Exocrine pancreatic insufficiency in diabetic patients: Prevalence, mechanisms, and treatment. Int J Endocrinol. 2015;2015:595649.

31. Andersen DK, Korc M, Petersen GM, Eibl G, Li D, Rickels MR, et al. Diabetes, pancreatogenic diabetes, and pancreatic cancer. Diabetes. 2017;66(5):1103-10.

32. Fridlyand LE, Philipson LH. Does the glucose-dependent insulin secretion mechanism itself cause oxidative stress in pancreatic $\beta$-cells? Diabetes. 2004;53:1942-8.

33. Forouhi NG, Harding AH, Allison M, Sandhu MS, Welch A, Luben R, et al. Elevated serum ferritin levels predict new-onset type 2 diabetes: results from the EPIC-Norfolk prospective study. Diabetologia. 2007; 50:949e956.

34. Lu JP, Hayashi K. Selective iron deposition in pancreatic islet B cells of transfusional iron-overloaded autopsy cases. Pathol Int. 1994;44: 194-9.

35. Simcox JA, McClain DA. Iron and diabetes risk. Cell Metab. 2013;17: 329-41.

36. Sun L, Zong G, Pan A, Ye X, Li H, Yu Z, et al. Elevated plasma ferritin is associated with increased incidence of type 2 diabetes in middle-aged and elderly Chinese adults. J Nutr 2013;143:1459e1465. 